\section{Evidence for the Natural Radioactivity of Indium}

A consideration of the energetics of radioactive decay shows that two isotopes of the same mass number but neighbouring atomic number cannot both be stable if the rest mass of the neutrino is zero. A number of such neighbouring isobaric pairs exist; in Nature; in some cases the expected radioactivity has been found in one member of the pair, with a period of decay comparable to, or longer than, the age of the earth (for example, the well-known case of ${ }_{19}^{\circ} \mathrm{K}$ ). The two naturally existing isotopes of indium- ${ }_{49}^{113} \mathrm{In}$ and ${ }_{45}^{115} \mathrm{In}$ (abundances 4.5 and 94.5 per cent respectively) --are each members of such isobaric pairs, the first with ${ }_{48}^{113} \mathrm{Cd}$, and the second with ${ }_{50}^{115} \mathrm{Sn}$. Accordingly, it was decided to search for radioactivity among these elements, as none had hithorto been found.

Positive results were obtained with indium. Using Geiger counters with easily changeable cathodes, a definite increase in counting-rate above the background-rate was detected when indium cathodes were used. The increase was of the order of 15 pulses per minute from a cathode of effective area $75 \mathrm{sq} . \mathrm{cm}$. and thickness $100 \mathrm{mgm} . / \mathrm{cm} .^{2}$. Indium samples of different origin and of spectrographic purity were used and gave consistent results. The possibility that the counts were due to $\alpha$-particle contamination was excluded by the results obtained using proportional counters and from absorption measurements.

I first tried to find if the radiation from indium could be identified with the characteristic $K \mathrm{X}$-rays of cadmium, which would be expected to follow the decay of ${ }_{49}^{113} \mathrm{In}$ to ${ }_{48}^{113} \mathrm{Cd}$ by $K$-orbital electron capture. The experiments were carried out with krypton-filled counters which had an efficiency nearly unity for these $\mathrm{X}$-rays. The results were negative, and showed (in agreement with the results of previous workers ${ }^{1}$ ) that the half-life for the decay of ${ }_{49}^{113} \mathrm{In}$ to ${ }_{48}^{113} \mathrm{Cd}$ by $K$-capture must be greater than $10^{14}$ years.

There remain two possibilities: first, the decay of ${ }_{49}^{115} \mathrm{In}$ to ${ }_{50}^{115} \mathrm{Sn}$ by $\beta$-emission; secondly, the decay of ${ }_{49}^{413} \mathrm{In}$ to ${ }_{48}^{113} \mathrm{Cd}$ by $L$-orbital electron capture. Most of the radiation, in fact, was found to consist of $\beta$-rays. The half-value absorption thickness through aluminium under bad geometry was found to be about $10 \mathrm{mgm} . / \mathrm{cm} .^{2}$, indicating that the energy of the $\beta$-rays must be of the order of a few hundred kilovolts. The interpretation of these results would appear to be that ${ }_{40}^{115} \mathrm{In}$ decays by $\beta$-emission to ${ }_{50}^{115 \mathrm{Sn}}$ with a half-life of the order of $10^{14}$ years.

The second possibility - that part of the radiation may be $L$ X-rays following the decay of ${ }_{49}^{113} \mathrm{In}$ by $L$-capture-is of considerable interest. Outer electron capture should always accompany $K$-capture ; but, in general, only to a small extent. It is consequently very difficult to detect, and, in fact, has only been observed in the case of ${ }_{18}^{37} \mathrm{~A}$, using proportional counter technique ${ }^{2}$. In some exceptional circumstances, depending on the difference in energy and angular momentum between the two nuclei involved, it is possible that the probability of $L$-electron capture is greater than that of capture from the $K$-shell. Using counters with different gas fillings and hence different efficiencies for cadmium $L \mathrm{X}$-rays, results were obtained which were consistent with the hypothesis that a small part of the radiation consisted of cadmium $L \mathrm{X}$-rays ; but owing to the small countingrates involved, the results cannot be regarded as conclusive evidence for this process. Assuming
$L$-capture, one arrives at a half-life of the order of $10^{12}$ years for the decay of ${ }_{49}^{113} \mathrm{In}$ in this way.

In order to arrive at a moro conclusive result on the $L$-capture process, the use of indium trimethyl vapour as a filling in a proportional counter has been considered.

This work was carried out at the Cavondish Laboratory, Cambridge, during 1947 and 1948. I would like to express my thanks to Prof. O. R. Frisch for his encouragement of this study. A detailed account of the experiments will be published in the near future.

Addendum. After this note was written, I had the opportunity (through the kindness of Dr. J. V. Dunworth) of reading an account of more recent and independent work of Libby and Martell (unpublished), using indium samples with isotopic enrichment. Their results essentially confirm the $\beta$-radioactivity of ${ }_{49}^{115} \mathrm{In}$. No conclusive evidence for orbital capture in ${ }_{4,9}^{413}$ In has been found.

Department of Physics, Hebrew University, Jerusalem. Nov, 27.

${ }^{1}$ Russinow and Igelnitsky, C.R. Acad. Sci. U.R.S.S., 47, 333 (1945); 49, $343(1945)$.

${ }^{2}$ Kirkwood, Pontecorvo and Hanna, Phys. Rev., 74, 497 (1918).

\section{Lattice Vibrations and Optical Waves in Ionic Crystals}

LATTICE vibrations in ionic crystals are usually treated with the unretarded Coulomb forces. Despite some discussions in the existing literature ${ }^{1,2}$, the precise nature of the effect due to retardation remains unknown. We have found that explicit solution of the problem is possible for diatomic crystals with optical isotropy, mainly because the lattice vibrations concerned are very long waves, for which the lattice behaviours can be described by the following equations ${ }^{3}$ :

$$
\begin{gathered}
\ddot{w}=-\omega_{0}^{2} \mathbf{w}+\left(\frac{\varepsilon_{0}-\varepsilon_{\infty}}{4 \pi}\right)^{1 / 2} \omega_{0} \mathbf{E} ; \\
\mathbf{P}=\left(\frac{\varepsilon_{0}-\varepsilon_{\infty}}{4 \pi}\right)^{1 / 2} \omega_{0} \mathbf{w}+\left(\frac{\varepsilon_{\infty}-1}{4 \pi}\right) \mathbf{E} ;
\end{gathered}
$$

where $w$ is the displacement of the positive ions relative to the negative, multiplied by the square root of the ratio of the reduced mass of the ions to the lattice cell volume; $\mathbf{P}$ and $\mathbf{E}$ are the dielectric polarization and the electric field; $\varepsilon_{0}, \varepsilon_{\infty}$ and $\omega_{0}$ are the static and high-frequency dielectric constants and the infra-red dispersion frequency (circular).

As shown elsewhere ${ }^{3}$, if the electric field in the lattice is treated according to electrostatics (equivalent to unretarded forces) by considering the equations (1) and (2) in conjunction with

$$
\nabla \cdot(\mathbf{E}+4 \pi \mathbf{P})=0,
$$

one obtains the usual longitudinal $\left(\omega=\left(\varepsilon_{0} / \varepsilon_{\infty}\right)^{1 / 2} \omega_{0}\right)$ and transverse $\left(\omega=\omega_{0}\right)$ lattice modes ${ }^{4-6}$, which are represented by dotted lines in the $\omega \sim 1 / \lambda$ graph. In order to take account of the retardation effect, one has to bring in the remaining Maxwell equations :

$$
\begin{gathered}
\nabla \cdot \mathbf{H}=0 ; \\
\nabla \times \mathbf{E}=-\frac{1}{c} \dot{\mathbf{H}} ; \\
\nabla \times \mathbf{H}=\frac{1}{c}(\dot{\mathbf{E}}+4 \pi \dot{\mathbf{P}}) .
\end{gathered}
$$

These equations, we note, necessarily bring the radiation field into the picture as well. Consider the case when all the ions are held fixed in their equili- 